\title{
Infrared-Mediated Drug Elution Activity of Gold Nanorod-Grafted $\mathrm{TiO}_{2}$ Nanotubes
}

\author{
Kyung-Suk Moon, ${ }^{1}$ Ji-Myung Bae, ${ }^{1}$ Sungho Jin, ${ }^{2}$ and Seunghan Oh${ }^{1}$ \\ ${ }^{1}$ Department of Dental Biomaterials and Institute of Biomaterial and Implant, College of Dentistry, Wonkwang University, \\ 344-2 Shinyong dong, Iksan, Jeonbuk 570-749, Republic of Korea \\ ${ }^{2}$ Department of Mechanical and Aerospace Engineering, University of California, La Jolla, San Diego, CA 92093, USA
}

Correspondence should be addressed to Seunghan Oh; shoh@wku.ac.kr

Received 26 May 2014; Accepted 16 July 2014; Published 24 July 2014

Academic Editor: Young-Bum Park

Copyright (C) 2014 Kyung-Suk Moon et al. This is an open access article distributed under the Creative Commons Attribution License, which permits unrestricted use, distribution, and reproduction in any medium, provided the original work is properly cited.

\begin{abstract}
The purpose of this research was to prepare gold nanorod- (GNR-) grafted $\mathrm{TiO}_{2}$ nanotubes by thiolactic acid treatment and evaluate remote-controlled drug elution and antibacterial activity by infrared (IR) light irradiation. Tetracycline used as an antibiotic was loaded into GNR-grafted $\mathrm{TiO}_{2}$ nanotubes by using $2 \mathrm{w} / \mathrm{v} \%$ polylactic acid solutions. A near-IR laser ( $830 \mathrm{~nm}$ ) was used for remotecontrolled IR light irradiation. Results of SEM, TEM, XRD, and EDX revealed that GNR chemically bonded to the whole surface of the $\mathrm{TiO}_{2}$ nanotubes. An antibiotic release test revealed that on-off drug elution was triggered effectively by the photothermal effect of GNR grafted on $\mathrm{TiO}_{2}$ nanotubes. Furthermore, an antibacterial agar zone test indicated that the annihilated zone of Streptococcus mutans in the experimental group with IR light irradiation was significantly larger than that of the corresponding group without IR light irradiation $(P<0.05)$. Therefore, $\mathrm{GNR}_{\text {-grafted }} \mathrm{TiO}_{2}$ nanotubes would be expected to extend the limited usage of $\mathrm{TiO}_{2}$, which show photocatalytic activity only within the ultraviolet (UV) to IR region, thereby allowing the development of novel fusion technologies in the field of implant materials.
\end{abstract}

\section{Introduction}

The long-term clinical success of implants is decided by osseointegration at the interface of an implant and bone tissue at the early stage of implantation [1]. Many studies have been conducted on accelerating osseointegration by modifying the surface roughness of implants and coating them with growth factors or hormones, which enhances the initial attachment of bone marrow cells, on the basis of the chemical properties, charge, microstructure, and porosity of the implant surface [1-4]. However, most recent techniques for enhancing osseointegration are also vulnerable to bacterial infection. Bacteria are as likely to attach to a surface coated by growth factor or proteins, as bone marrow cells are. Once bacteria permeate into the coated surface, it is impossible to eradicate them by using common sterilization techniques.

The resolution of bacterial infection generally requires antibiotic therapy approximately 2 months after implant surgery. There are many ways to deliver antibiotics to a target in a patient's body, including systemic, intravenous, intramuscular, and topical methods. Systemic delivery is typically associated with some side effects as well as inevitable cytotoxicity. Much research has been undertaken to develop various drug delivery systems to achieve efficacy at a localized target area [5, 6].

Most of the recently developed drug delivery systems have excellent targeting capability and proper drug delivery and afford an immediate reduction of bacterial infection [711]. However, most of these systems do not afford control of the time and period of drug elution. In particular, drug elution is not required immediately after implant surgery, but 2 months after the surgery. Therefore, a new delivery system that affords control of the time of drug elution and accurate dosing of antibiotics to the target area is required.

$\mathrm{TiO}_{2}$ nanotubes have been studied predominantly in the field of photocatalysis [12-14], solar cells [15], and biomedical 
engineering [16-18], because of their excellent photocatalytic activity and biocompatibility. In addition, the high surface area and unique shape of $\mathrm{TiO}_{2}$ nanotubes make them a promising option for application in drug delivery systems.

$\mathrm{TiO}_{2}$ photocatalysts have been mostly used for the purification of air and water $[19,20]$ by UV light irradiation. However, the limited photocatalytic activity of $\mathrm{TiO}_{2}$ within the UV range presents difficulties in biomedical and tissue engineering applications owing to the potential harmful effects of UV light. Our previous study was focused on extension of the photocatalytic activity of $\mathrm{TiO}_{2}$ to the visible light region by doping nitrogen into the $\mathrm{TiO}_{2}$ crystal structure, which would allow remote control of antibiotic elution by visible light irradiation [21]. On the basis of the results of our previous study, we expected IR light to be more effective than visible light, owing to its superior skin penetration.

Gold nanorods (GNR) are known to have much potential for mediating photodynamic and photothermal effects at near IR light [22-27]. As such, GNR have found promising application in the field of cancer treatment as a photosensitizer. In other medical applications, IR light has been widely used for the therapy of back pain and chronic nonhealing wounds [28]. IR light can also overcome the limitation of visible light, which cannot penetrate deep into the skin. We anticipate that the photothermal activity of GNR would allow for remote control of on-off drug elution by IR laser irradiation.

In this study, we immobilized GNR at the surface of $\mathrm{TiO}_{2}$ nanotubes via a grafting technique and investigated on-off drug release triggered by near-IR laser irradiation. In addition, antimicrobial activity was monitored to assess the effectiveness of GNR-grafted $\mathrm{TiO}_{2}$ nanotubes in allowing remotely controlled drug release by IR laser irradiation.

\section{Materials and Methods}

2.1. Fabrication of $\mathrm{TiO}_{2}$ Nanotubes. As reported previously [29], a machined Ti sheet (0.2 mm thick, 99.5\%; Hyundai Titanium Co., South Korea) was polished by an electropolishing process and cleaned with ethyl alcohol (99.5\%, Dae-Jung Chemical, South Korea) and double-distilled water. $\mathrm{TiO}_{2}$ nanotubes (diameter; $100 \mathrm{~nm}$ ) were prepared in $0.5 \mathrm{w} / \mathrm{v} \%$ hydrofluoric acid (48 w/v\%, Merck, NJ, USA) in water with acetic acid ( $98 \mathrm{w} / \mathrm{v} \%$, JT Baker, NJ, USA; volumetric ratio = $7: 1)$ at $20 \mathrm{~V}$ for $1 \mathrm{~h}$. A platinum electrode (99.99\%, DSM Co., South Korea) served as the counterpart. The samples were then rinsed with double-distilled water, dried at $60^{\circ} \mathrm{C}$, and heat-treated at $400^{\circ} \mathrm{C}$ for $3 \mathrm{~h}$ to crystallize amorphous $\mathrm{TiO}_{2}$ nanotubes into anatase structures.

2.2. Gold Nanorod (GNR) Synthesis. GNR were synthesized by modifying the three-step protocol described previously [30]. First, a solution was prepared to facilitate the seeding of GNR. $5 \mathrm{~mL}$ of $0.0005 \mathrm{M} \mathrm{HAuCl}_{4}$ (Sigma, MO, USA) and $60 \mu \mathrm{L}$ of $0.01 \mathrm{M} \mathrm{NaBH}_{4}$ (Sigma, MO, USA) were mixed vigorously in $5 \mathrm{~mL}$ of $0.2 \mathrm{M}$ cetyltrimethylammonium bromide (CTAB; Sigma, MO, USA) for 2 min. The mixed seed solution was stored at $25^{\circ} \mathrm{C}$ and used within $3 \mathrm{~h}$. Secondly, another solution was prepared to grow crystals from the GNR seeds. $200 \mu \mathrm{L}$ of $0.004 \mathrm{M} \mathrm{AgNO}_{3}$ (Sigma, MO, USA) and $500 \mu \mathrm{L}$ of $0.01 \mathrm{M} \mathrm{HAuCl}_{4}$ were added to $5 \mathrm{~mL}$ of $0.2 \mathrm{M} \mathrm{CTAB}$ solution and mixed until the solution turned yellow. Further addition of $70 \mu \mathrm{L}$ of $0.0788 \mathrm{M} \mathrm{L}$-ascorbic acid (Sigma, MO, USA) discharged the color, leading to a clear solution. $12 \mu \mathrm{L}$ of the previously prepared seed solution was then added to this solution, and the mixture was shaken gently and stored at $25^{\circ} \mathrm{C}$ for $4 \mathrm{~h}$.

2.3. GNR Grafting Process. To graft GNR at the surface of $\mathrm{TiO}_{2}$ nanotubes, we modified the protocol reported previously $[31,32]$. As shown in Figure $1, \mathrm{TiO}_{2}$ nanotubes were first treated with various concentrations of aqueous thiolactic acid (0.1, 0.2, and 0.5 M; Sigma, MO, USA) for $30 \mathrm{~min}$ and then dried at $60^{\circ} \mathrm{C}$ for $12 \mathrm{~h}$. The GNR solution prepared above was added to the pretreated $\mathrm{TiO}_{2}$ nanotubes, and the mixture was stored at $25^{\circ} \mathrm{C}$ for $2 \mathrm{~h}$ and then dried at $60^{\circ} \mathrm{C}$ for $12 \mathrm{~h}$.

2.4. Surface Characterization. The morphology and optical properties of synthesized GNR were assessed by transmission electron microscopy (TEM; JEM-2010, JEOL, Japan) and UV-Vis spectrophotometry (UVmini-1240, Shimadzu, Japan). Morphologic observation and crystal structure of GNR-grafted $\mathrm{TiO}_{2}$ nanotubes were determined using a field emission scanning electron microscope (FE-SEM; S4800, Hitachi/Horiba Co., Japan) and thin film X-ray diffractometer (XRD; X'PERT-MRD, Phillips Co., USA), respectively. The amount of GNR grafted at the surface of $\mathrm{TiO}_{2}$ nanotubes was analyzed by energy dispersive X-ray spectroscopy (EDX) equipped with FE-SEM.

2.5. Drug Release Test. A solution of $10 \mathrm{~g}$ of tetracycline (Sigma, MO, USA) in $50 \mathrm{~mL}$ of DMSO was added to a solution of $4 \mathrm{~g}$ of polylactic acid (PLA; Sigma, MO, USA) in $50 \mathrm{~mL}$ of tetrahydrofuran (THF; Sigma, MO, USA) and the mixture was stirred for $3 \mathrm{~h}$. The resulting solution was loaded into $\mathrm{TiO}_{2}$ nanotubes by a vacuum loading technique [21]. A microplate ELISA reader (Spectra Max 250; Thermo Electron Co., USA) measured the amount of tetracycline released as a function of incubation period. The amount of tetracycline released in response to IR light irradiation was also measured by a microplate ELISA reader. The source of IR light was a near-IR laser (Power: $200 \mathrm{~mW}$ and wavelength: $830 \mathrm{~nm}$; RaeHwa LMA, South Korea).

2.6. Antibacterial Agar Diffusion Test. To perform the antibacterial agar diffusion test of GNR-grafted $\mathrm{TiO}_{2}$ nanotubes, Streptococcus mutans (ATCC 25176, ATCC, USA) was incubated in brain-heart infusion broth (BHIB; Difco Co., USA). When the bacterium showed high confluence, $100 \mu \mathrm{L}$ of the incubating solution was diluted into $1 \times$ $10^{5} \mathrm{CFU} / \mathrm{mL}$ and seeded onto agar medium. The combination was stored in an incubator at $37^{\circ} \mathrm{C}$ for $24 \mathrm{~h}$ in order to solidify the agar medium and form the Streptococcus mutans colony. Experimental specimens $\left(1 \times 1 \mathrm{~cm}^{2}\right)$ were placed onto Streptococcus mutans incubated solid agar medium, and IR laser irradiation was conducted for $1 \mathrm{~min}$. After IR laser 

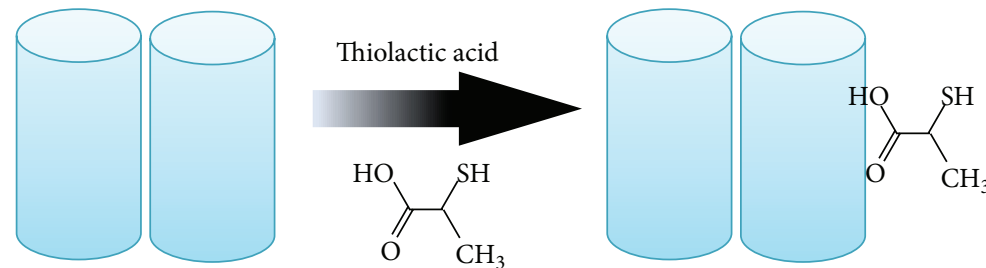

Gold nanorod

(GNR)
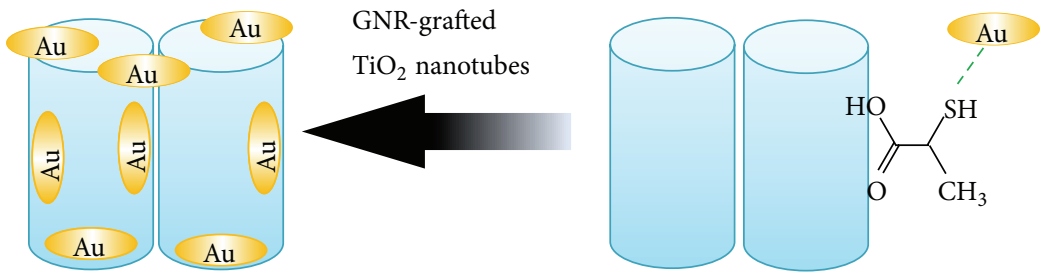

FIGURE 1: Schematic diagram of preparing gold nanorod- (GNR-) grafted $\mathrm{TiO}_{2}$ nanotubes by thiolactic acid treatment.

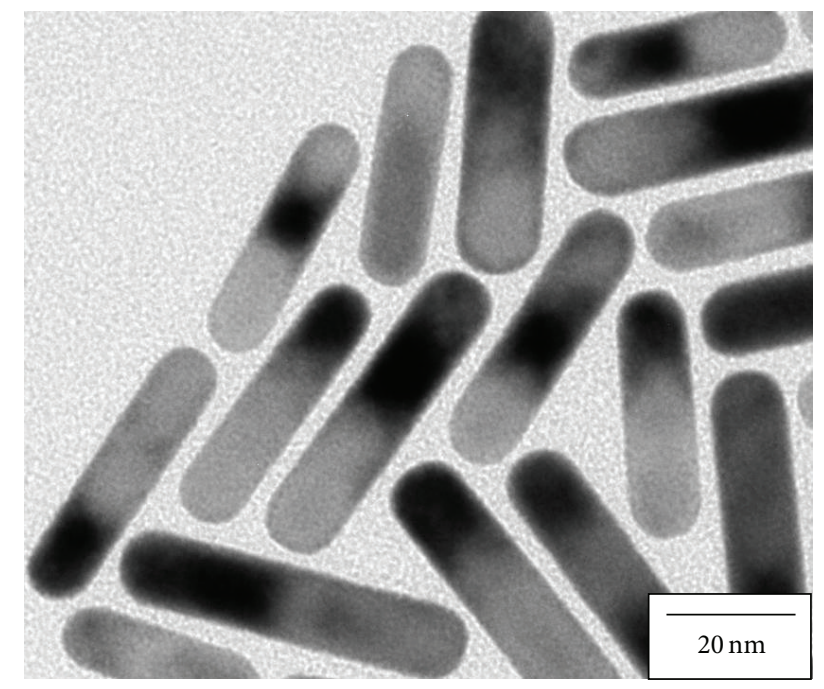

(a)

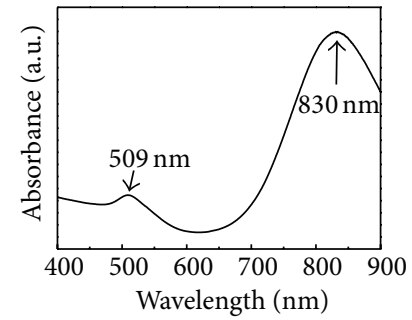

(b)

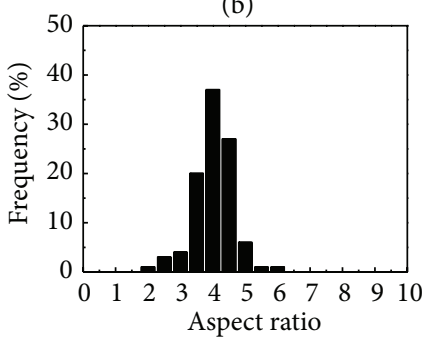

(c)

FIGURE 2: (a) TEM micrograph and (b) UV-Vis absorption spectra of GNR prepared in this research. (c) The distribution of GNR aspect ratio obtained from TEM micrographs.

irradiation, the specimen was stored at $37^{\circ} \mathrm{C}$ for $24 \mathrm{~h}$. The experimental specimens without IR laser treatment were placed directly at $37^{\circ} \mathrm{C}$ incubator and stored for $24 \mathrm{~h}$. After a second incubation period of 24 hours, the zone diameters of the death of the Streptococcus mutans colony in control and experimental specimens were measured 3 times per specimen in order to minimize the error of measurement.

2.7. Data Analysis. All data were expressed as mean \pm standard deviation values and analyzed statistically by oneway ANOVA (SPSS 12.0; SPSS GmbH, Germany) and post hoc Duncan's multiple range tests. Significant differences were considered if $P$ values were less than 0.05 .

\section{Results and Discussion}

Figure 2 shows the TEM micrograph and UV-Vis spectrum results of GNR synthesized in this study. From the TEM image and the aspect ratio distribution diagram of GNR, relatively uniform-sized GNR (diameter; $10 \pm 1 \mathrm{~nm}$, aspect ratio; 3.83) were synthesized, and round-shaped gold nanoparticles were not detected. The electron absorption spectrum of synthesized GNR showed two peaks at wavelength of 509 and $830 \mathrm{~nm}$, which was the main phenomenon of photothermal scattering of short and long axis of GNR, respectively. In this study, $830 \mathrm{~nm}$ near-IR laser was used to perform onoff drug release and remote-controlled antimicrobial activity 


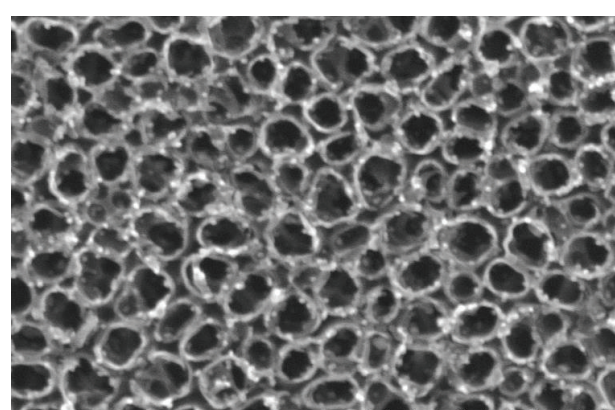

(a)

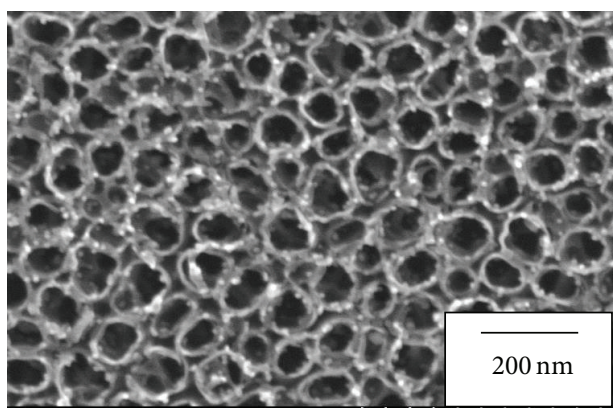

(c)

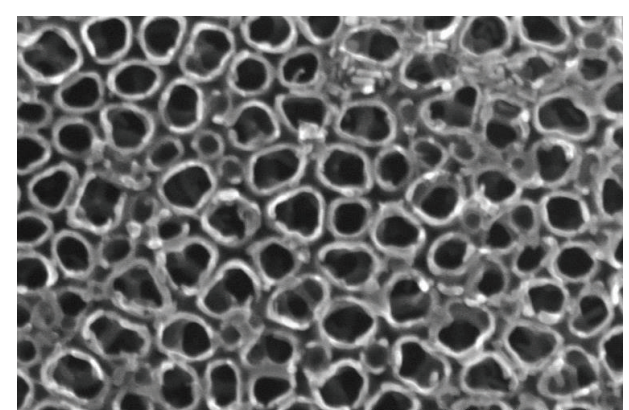

(b)

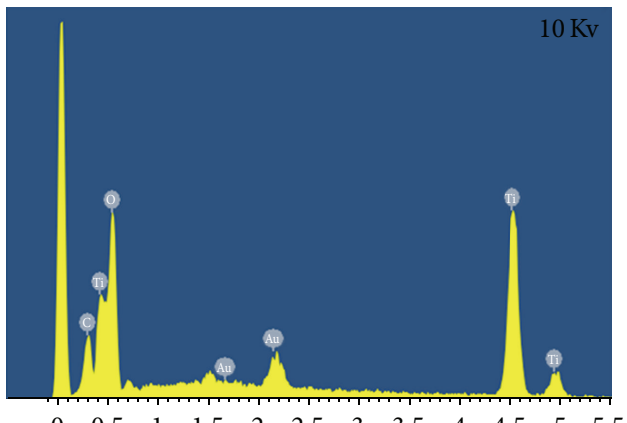

$\begin{array}{llllllllllll}0 & 0.5 & 1 & 1.5 & 2 & 2.5 & 3 & 3.5 & 4 & 4.5 & 5 & 5.5\end{array}$

(d)

Figure 3: ((a), (b), (c)) FE-SEM micrographs of GNR-grafted $\mathrm{TiO}_{2}$ nanotubes treated by (a) $0.1 \mathrm{M}$, (b) $0.5 \mathrm{M}$, and (c) $1.0 \mathrm{M}$ thiolactic acid solution. (d) EDX results of GNR-grafted $\mathrm{TiO}_{2}$ nanotubes treated by $0.1 \mathrm{M}$ thiolactic acid solution.

TABLE 1: EDX results of GNR-grafted $\mathrm{TiO}_{2}$ nanotubes treated by various concentrations of aqueous thiolactic acid solution.

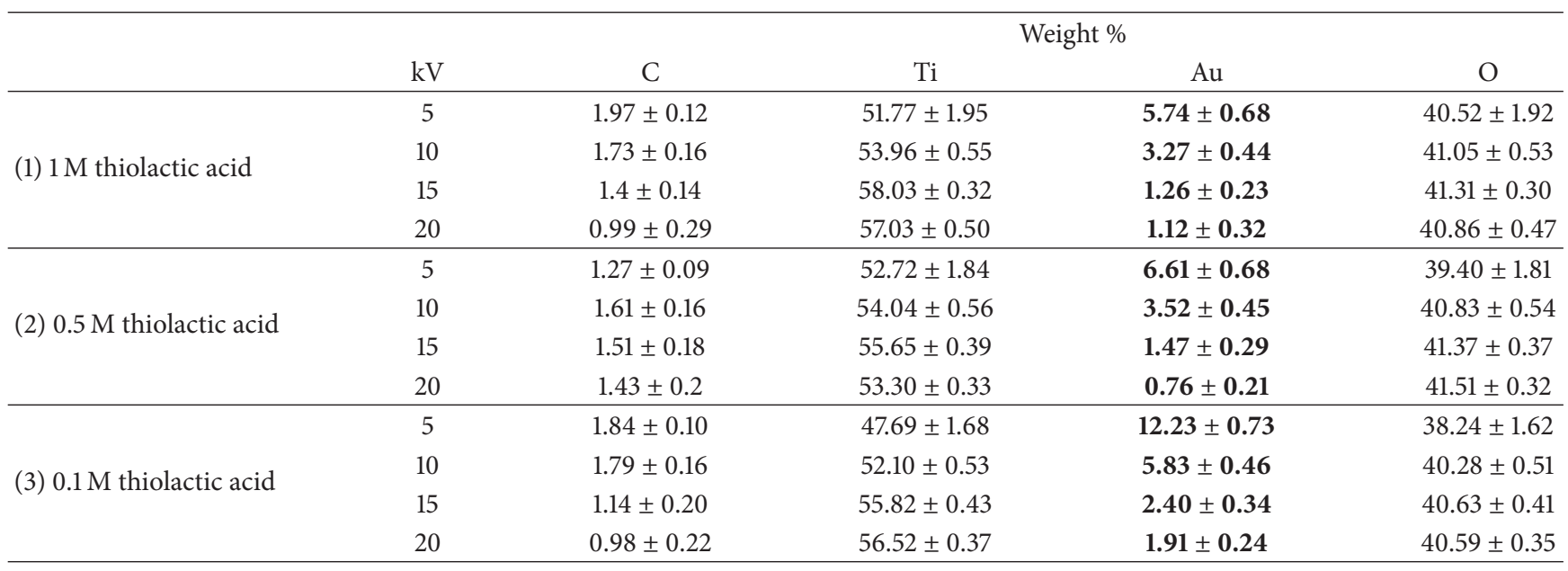

tests, thereby maximizing the photothermal effect of the synthesized GNR.

Figure 3 shows FE-SEM and EDX micrographs of GNRgrafted $\mathrm{TiO}_{2}$ nanotubes treated by different concentrations of thiolactic acid $(0.1,0.5$, and $1.0 \mathrm{M})$. As shown in the FESEM images, there was no destruction or blocking of $\mathrm{TiO}_{2}$ nanotubes after thiolactic acid treatment with different concentrations. In addition, small nanomaterials were observed on $\mathrm{TiO}_{2}$ nanotubes, but it is difficult to confirm whether these nanomaterials were GNR or not. From the EDX result, GNR chemically conjugated on $\mathrm{TiO}_{2}$ nanotubes were confirmed by the element analysis of Au.
Table 1 lists the amounts of main elements detected at the surface of $\mathrm{TiO}_{2}$ nanotubes treated by different concentrations of aqueous thiolactic acid solution. From the results of elementary analysis, the total amount of gold detected by EDX was less than $12 \mathrm{wt} \%$. In addition, $0.1 \mathrm{M}$ thiolactic acid showed the most effective grafting among the three thiolactic acid concentrations. To double-check the optimal concentration of thiolactic acid solution, we tested elementary analysis on the conditions of 0.01 and $0.05 \mathrm{M}$ thiolactic acid solution and confirmed that the gold contents of $0.1 \mathrm{M}$ thiolactic acid treated groups were higher than that of 0.01 and $0.05 \mathrm{M}$ thiolactic acid treated group (data are not shown). 


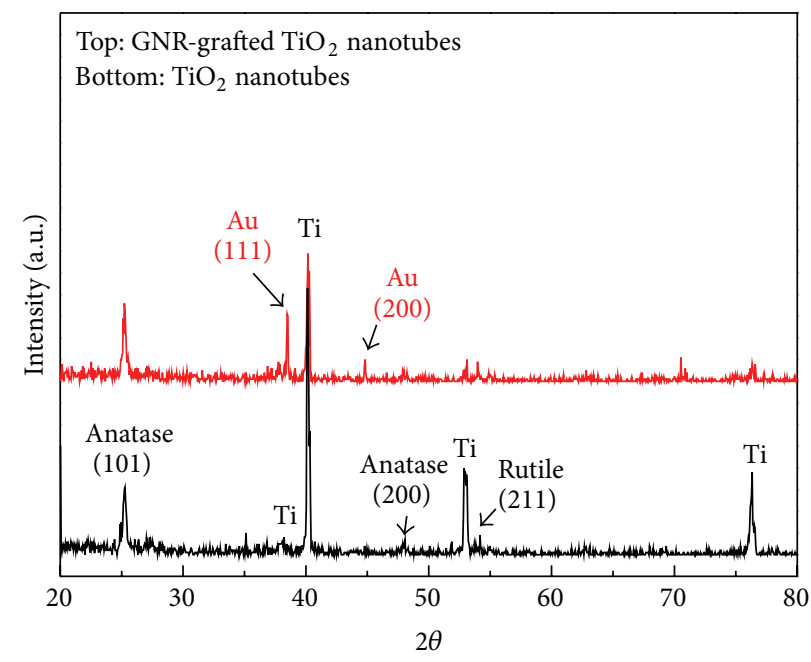

FIGURE 4: X-ray diffraction patterns of GNR-grafted $100 \mathrm{~nm} \mathrm{TiO}{ }_{2}$ nanotubes treated by $0.1 \mathrm{M}$ thiolactic acid.

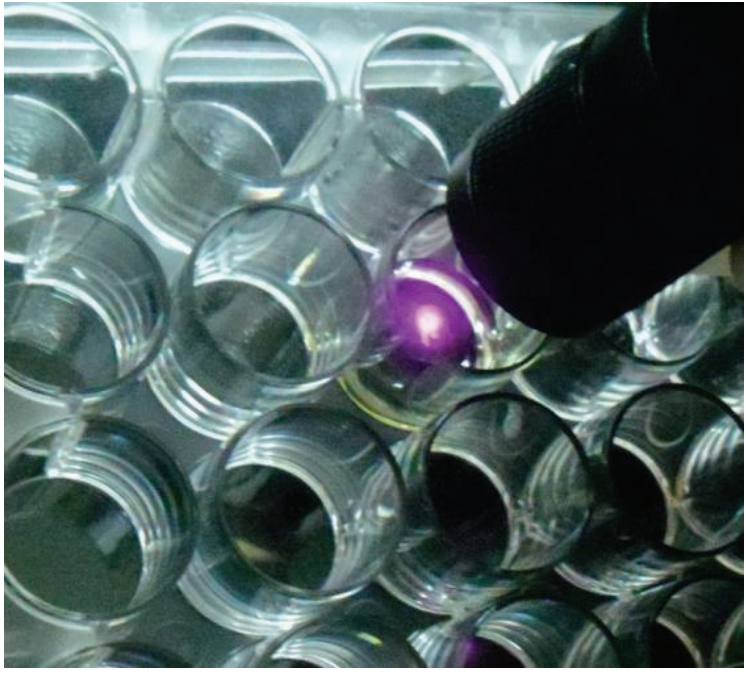

(a)

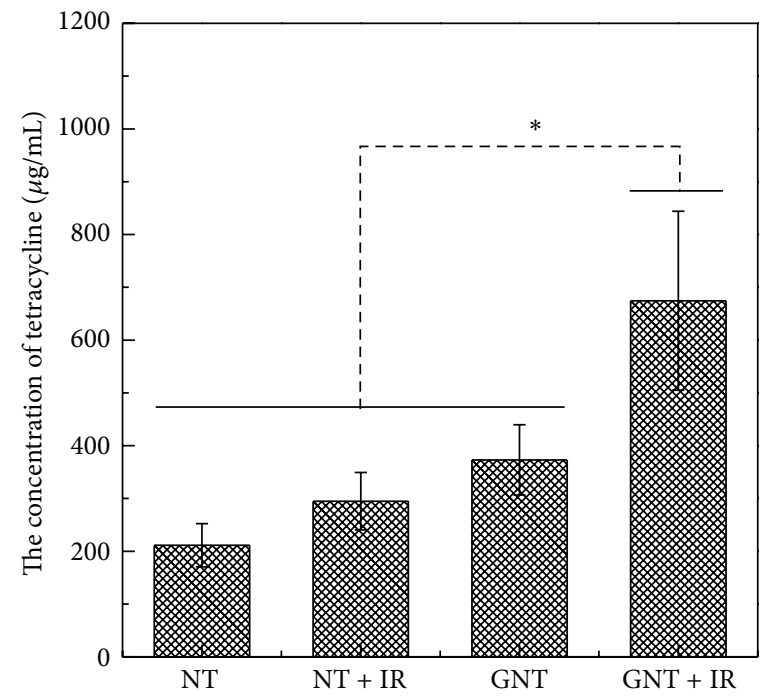

(b)

FIGURE 5: (a) Near-IR laser and IR light irradiation and (b) the concentration of tetracycline released by IR light irradiation (NT, NT + IR, GNT, and GNT + IR in the graph designate $\mathrm{TiO}_{2}$ nanotubes only, near-IR laser irradiated $\mathrm{TiO}_{2}$ nanotubes, $\mathrm{GNR}_{\text {-grafted }} \mathrm{TiO}{ }_{2}$ nanotubes, and near-IR laser irradiated GNR grafted $\mathrm{TiO}_{2}$ nanotubes, resp.). $*$ denotes significance between groups $(P<0.05)$.

Figure 4 displays X-ray diffraction (XRD) patterns of GNR-grafted $\mathrm{TiO}_{2}$ nanotubes. As shown, XRD mainly detected anatase $\mathrm{TiO}_{2}$ and $\mathrm{Ti}$ crystalline phases from the XRD patterns of $\mathrm{TiO}_{2}$ nanotubes. In addition, the XRD pattern of GNR-grafted $\mathrm{TiO}_{2}$ nanotubes indicated that two additional peaks corresponding to the (111) and (200) peaks of gold nanorods were detected at 38 and $44.8^{\circ}$, respectively, as reported previously [33]. Therefore, we concluded that the covalent immobilization of GNR had no effect on the crystallinity of $\mathrm{TiO}_{2}$ nanotubes in this study, and XRD measurement was enough to detect gold deposited on $\mathrm{TiO}_{2}$ nanotubes, even though the grafted amounts of GNR were small as shown by the results of elementary analysis (EDX).
Figure 5 shows the elution concentrations of tetracycline loaded at the surface of GNR-grafted $\mathrm{TiO}_{2}$ nanotubes after $30 \mathrm{~s}$ of IR light irradiation with a near-IR laser. The release concentrations of tetracycline from GNR-grafted $\mathrm{TiO}_{2}$ nanotubes with IR light irradiation $(674.52 \pm 169.58 \mu \mathrm{g} / \mathrm{mL})$ were significantly higher than those from other experimental conditions, such as $\mathrm{TiO}_{2}$ nanotubes with or without IR light irradiation and GNR-grafted $\mathrm{TiO}_{2}$ nanotubes without IR laser irradiation $(P<0.05)$.

Figure 6 demonstrates the zone index results of antibacterial agar diffusion test with GNR-grafted $\mathrm{TiO}_{2}$ nanotubes after $1 \mathrm{~min}$ of IR light irradiation with a near-IR laser. In this examination, negative (sterilized filter paper only) and 

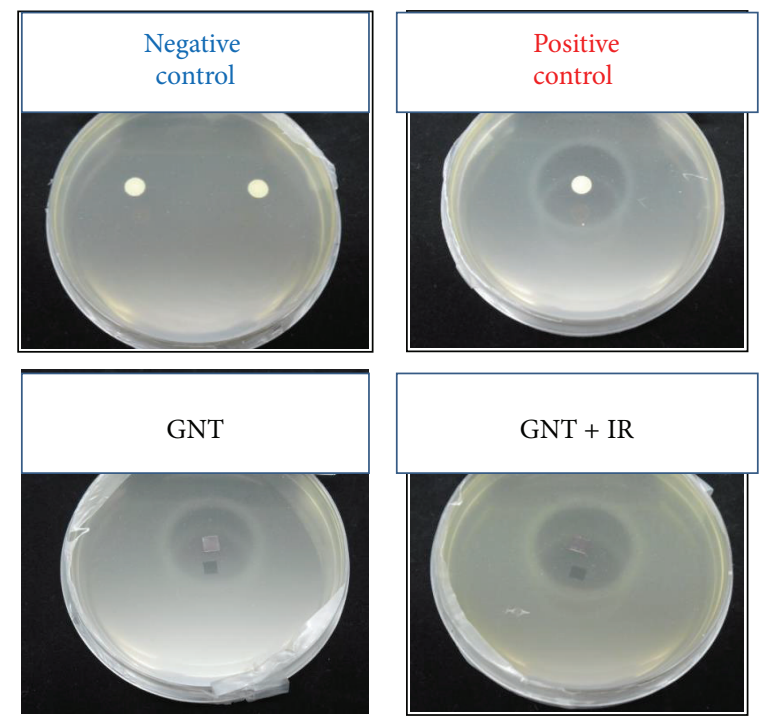

(a)

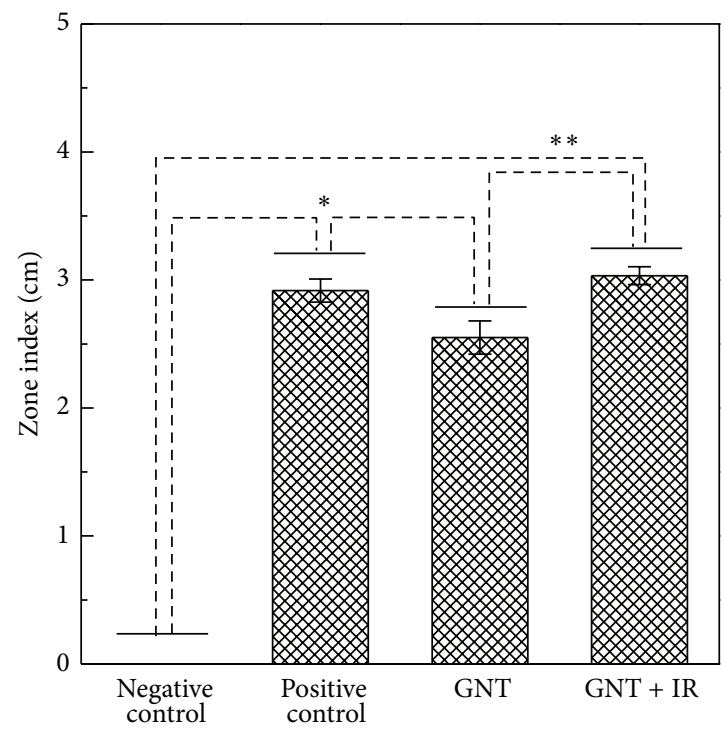

(b)

Figure 6: (a) Photographs of antibacterial agar diffusion test by using Streptococcus mutans and (b) the results of zone diameter of dead Streptococcus mutans on the conditions of negative control, positive control, GNR-grafted $\mathrm{TiO}_{2}$ nanotubes without IR light irradiation (designated GNT) and with IR light irradiation for 1 min (designated GNT + IR). * and $* *$ denote significance between groups $(P<0.05)$.

positive ( $3 \mu \mathrm{L}$ tetracycline loaded filter paper) control groups were adopted to compare the values of experimental groups. The zone index of annihilated Streptococcus mutans of GNRgrafted $\mathrm{TiO}_{2}$ nanotubes with IR light irradiation (3.03 \pm $0.07 \mathrm{~cm}$ ) was significantly higher than that of GNR-grafted $\mathrm{TiO}_{2}$ nanotubes without IR light irradiation $(2.55 \pm 0.13 \mathrm{~cm})$ $(P<0.05)$. In addition, GNR-grafted $\mathrm{TiO}_{2}$ nanotubes without IR light irradiation showed the annihilation effect of Streptococcus mutans regardless of IR light irradiation. This phenomenon is supposed to be the long period of second incubation after plating experimental specimen. GNR-grafted $\mathrm{TiO}_{2}$ nanotubes including the mixture of PLA and tetracycline were contacted with Streptococcus mutans during second incubation period. Therefore, $24 \mathrm{~h}$ of second incubation is seemed to be enough to kill the bacterium, even though the release amount of tetracycline is small. Further investigation will be performed to clarify on-off drug release remotely controlled by IR light irradiation.

Rod-shaped GNR are well known to mediate photodynamic effects at near infrared light and tune the surface plasmon resonance (SPR) band from the visible to near IR wavelength by modifying the aspect ratio of GNR [22, 34-36]. In addition to photodynamic cancer therapy, the nanomaterial-mediated photothermal therapy of GNR plays an important role in combining the therapeutic effect with photodynamic therapy to achieve cancer treatment. The main goal of this research was to detach antibiotics deposited on $\mathrm{TiO}_{2}$ nanotubes by IR laser irradiation based on the photodynamic and photothermal effects of GNR. In particular, the photothermal effect of GNR was expected predominantly to affect elution of drugs from $\mathrm{TiO}_{2}$ nanotubes remotely by nearIR laser.

\section{Conclusions}

From the results of this research, we can surmise that covalent immobilization of GNR on $\mathrm{TiO}_{2}$ nanotubes via a grafting technique was performed successfully by thiolactic acid treatment. Moreover, we confirm that tetracycline stored in GNR-grafted $\mathrm{TiO}_{2}$ nanotubes was released effectively by IR light irradiation with a near-IR laser, even though the amount of gold grafted at the surface of $\mathrm{TiO}_{2}$ nanotubes was less than $12 \mathrm{wt} \%$. Therefore, we conclude that GNR-grafted $\mathrm{TiO}_{2}$ nanotubes can be expected to extend the limited usage of $\mathrm{TiO}_{2}$ showing photocatalytic activity within the UV to IR region, thereby facilitating the development of novel fusion technologies in the field of implantation materials.

\section{Conflict of Interests}

The authors declare that there is no conflict of interests regarding the publication of this paper.

\section{Acknowledgment}

This research was supported by the Basic Science Research Program through the National Research Foundation of Korea (NRF) funded by the Ministry of Education, Science and Technology (2011-0024067). 


\section{References}

[1] L. Le Guéhennec, A. Soueidan, P. Layrolle, and Y. Amouriq, "Surface treatments of titanium dental implants for rapid osseointegration," Dental Materials, vol. 23, no. 7, pp. 844-854, 2007.

[2] R. Junker, A. Dimakis, M. Thoneick, and J. A. Jansen, "Effects of implant surface coatings and composition on bone integration: a systematic review," Clinical Oral Implants Research, vol. 20, no. 4, pp. 185-206, 2009.

[3] D. A. Puleo and A. Nanci, "Understanding and controlling the bone-implant interface," Biomaterials, vol. 20, no. 23-24, pp. 2311-2321, 1999.

[4] M. Dettin, M. T. Conconi, R. Gambaretto et al., "Effect of synthetic peptides on osteoblast adhesion," Biomaterials, vol. 26, no. 22, pp. 4507-4515, 2005.

[5] D. I. Axel, W. Kunert, C. Göggelmann et al., "Paclitaxel inhibits arterial smooth muscle cell proliferation and migration in vitro and in vivo using local drug delivery," Circulation, vol. 96, no. 2, pp. 636-645, 1997.

[6] G. Greenstein and A. Polson, "The role of local drug delivery in the management of periodontal diseases: a comprehensive review," Journal of Periodontology, vol. 69, no. 5, pp. 507-520, 1998.

[7] S. R. Naik, S. K. Desai, P. D. Shah, and S. M. Wala, "Liposomes as potential carrier system for targeted delivery of polyene antibiotics," Recent Patents on Inflammation \& Allergy Drug Discovery, vol. 7, no. 3, pp. 202-214, 2013.

[8] A. J. Sawyer, D. Wesolowski, N. Gandotra et al., "A peptidemorpholino oligomer conjugate targeting Staphylococcus aureus gyrA mRNA improves healing in an infected mouse cutaneous wound model," International Journal of Pharmaceutics, 2013.

[9] G. Gao and J. Xu, "Important biology events and pathways in Brucella infection and implications for novel antibiotic drug targets," Critical Reviews in Eukaryotic Gene Expression, vol. 23, no. 1, pp. 65-76, 2013.

[10] K. Gulati, M. S. Aw, and D. Losic, "Drug-eluting Ti wires with titania nanotube arrays for bone fixation and reduced bone infection," Nanoscale Research Letters, vol. 6, article 571, 2011.

[11] S. Liu, T. H. Bugge, and S. H. Leppla, "Targeting of tumor cells by cell surface urokinase plasminogen activator-dependent anthrax toxin," The Journal of Biological Chemistry, vol. 276, no. 21, pp. 17976-17984, 2001.

[12] S. Liu and A. Chen, "Coadsorption of horseradish peroxidase with thionine on $\mathrm{TiO}_{2}$ nanotubes for biosensing," Langmuir, vol. 21, no. 18, pp. 8409-8413, 2005.

[13] M. Paulose, K. Shankar, S. Yoriya et al., "Anodic growth of highly ordered $\mathrm{TiO}_{2}$ nanotube arrays to $134 \mu \mathrm{m}$ in length," Journal of Physical Chemistry B, vol. 110, no. 33, pp. 16179-16184, 2006.

[14] H. Zhang, P. Liu, X. Liu et al., "Fabrication of highly ordered $\mathrm{TiO}_{2}$ nanorod/nanotube adjacent arrays for photoelectrochemical applications," Langmuir, vol. 26, no. 13, pp. 11226-11232, 2010.

[15] K. Shankar, G. K. Mor, H. E. Prakasam, O. K. Varghese, and C. A. Grimes, "Self-assembled hybrid polymer- $\mathrm{TiO}_{2}$ nanotube array heterojunction solar cells," Langmuir, vol. 23, no. 24, pp. 1244512449, 2007.

[16] M. Bigerelle, K. Anselme, B. Noël, I. Ruderman, P. Hardouin, and A. Iost, "Improvement in the morphology of Ti-based surfaces: a new process to increase in vitro human osteoblast response," Biomaterials, vol. 23, no. 7, pp. 1563-1577, 2002.
[17] B. D. Boyan, T. W. Hummert, D. D. Dean, and Z. Schwartz, "Role of material surfaces in regulating bone and cartilage cell response," Biomaterials, vol. 17, no. 2, pp. 137-146, 1996.

[18] C. G. Galbraith and M. P. Sheetz, "Forces on adhesive contacts affect cell function," Current Opinion in Cell Biology, vol. 10, no. 5, pp. 566-571, 1998.

[19] A. L. Linsebigler, G. Lu, and J. T. Yates Jr., "Photocatalysis on $\mathrm{TiO}_{2}$ surfaces: principles, mechanisms, and selected results," Chemical Reviews, vol. 95, no. 3, pp. 735-758, 1995.

[20] M. R. Hoffmann, S. T. Martin, W. Choi, and D. W. Bahnemann, "Environmental applications of semiconductor photocatalysis," Chemical Reviews, vol. 95, no. 1, pp. 69-96, 1995.

[21] S. Oh, K. S. Moon, J. H. Moon, J. M. Bae, and S. Jin, "Visible light irradiation-mediated drug elution activity of nitrogen-doped $\mathrm{TiO}_{2}$ nanotubes," Journal of Nanomaterials, vol. 2013, Article ID 802318, 7 pages, 2013.

[22] R. Chen, X. Wang, X. Yao, X. Zheng, J. Wang, and X. Jiang, "Near-IR-triggered photothermal/photodynamic dualmodality therapy system via chitosan hybrid nanospheres," Biomaterials, vol. 34, no. 33, pp. 8314-8322, 2013.

[23] P. P. Joshi, S. J. Yoon, Y. S. Chen, S. Emelianov, and K. V. Sokolov, "Development and optimization of near-IR contrast agents for immune cell tracking," Biomedical Optics Express, vol. 4, no. 11, pp. 2609-2618, 2013.

[24] J. Choi, J. Yang, D. Bang et al., “Targetable gold nanorods for epithelial cancer therapy guided by near-IR absorption imaging," Small, vol. 8, no. 5, pp. 746-753, 2012.

[25] J. T. Robinson, K. Welsher, S. M. Tabakman et al., "High performance in vivo near-IR $(>1 \mu \mathrm{m})$ imaging and photothermal cancer therapy with carbon nanotubes," Nano Research, vol. 3, no. 11, pp. 779-793, 2010.

[26] T. Sada, T. Fujigaya, Y. Niidome, K. Nakazawa, and N. Nakashima, "Near-IR laser-triggered target cell collection using a carbon nanotube-based cell-cultured substrate," ACS Nano, vol. 5, no. 6, pp. 4414-4421, 2011.

[27] R. Guo, L. Zhang, H. Qian, R. Li, X. Jiang, and B. Liu, "Multifunctional nanocarriers for cell imaging, drug delivery, and near-IR photothermal therapy," Langmuir, vol. 26, no. 8, pp. 5428-5434, 2010.

[28] Z. Landau, M. Migdal, A. Lipovsky, and R. Lubart, "Visible light-induced healing of diabetic or venous foot ulcers: a placebo-controlled double-blind study," Photomedicine and Laser Surgery, vol. 29, no. 6, pp. 399-404, 2011.

[29] S. Yeonmi and L. Seonghoon, "Self-organized regular arrays of anodic $\mathrm{TiO}_{2}$ nanotubes," Nano Letters, vol. 8, no. 10, pp. 31713173, 2008.

[30] H. Liao and J. H. Hafner, "Gold nanorod bioconjugates," Chemistry of Materials, vol. 17, no. 18, pp. 4636-4641, 2005.

[31] C. Ratanatawanate, A. Chyao, and K. J. Balkus, "Snitrosocysteine-decorated $\mathrm{PbS} \quad \mathrm{QDs} / \mathrm{TiO}_{2}$ nanotubes for enhanced production of singlet oxygen," Journal of the American Chemical Society, vol. 133, no. 10, pp. 3492-3497, 2011.

[32] C. Ratanatawanate, C. Xiong, and K. J. Balkus Jr., "Fabrication of $\mathrm{PbS}$ quantum dot doped $\mathrm{TiO}_{2}$ nanotubes," ACS Nano, vol. 2, no. 8, pp. 1682-1688, 2008.

[33] V. Sebastián, S. K. Lee, C. Zhou, M. F. Kraus, J. G. Fujimoto, and K. F. Jensen, "One-step continuous synthesis of biocompatible gold nanorods for optical coherence tomography," Chemical Communications, vol. 48, no. 53, pp. 6654-6656, 2012.

[34] B. Wang, J. H. Wang, Q. Liu et al., "Rose-bengal-conjugated gold nanorods for in vivo photodynamic and photothermal oral 
cancer therapies," Biomaterials, vol. 35, no. 6, pp. 1954-1966, 2014.

[35] B. Jang, J. Park, C. Tung, I. Kim, and Y. Choi, "Gold nanorodphotosensitizer complex for near-infrared fluorescence imaging and photodynamic/photothermal therapy in vivo," ACS Nano, vol. 5, no. 2, pp. 1086-1094, 2011.

[36] S. Jelveh and D. B. Chithrani, "Gold nanostructures as a platform for combinational therapy in future cancer therapeutics," Cancers, vol. 3, no. 1, pp. 1081-1110, 2011. 

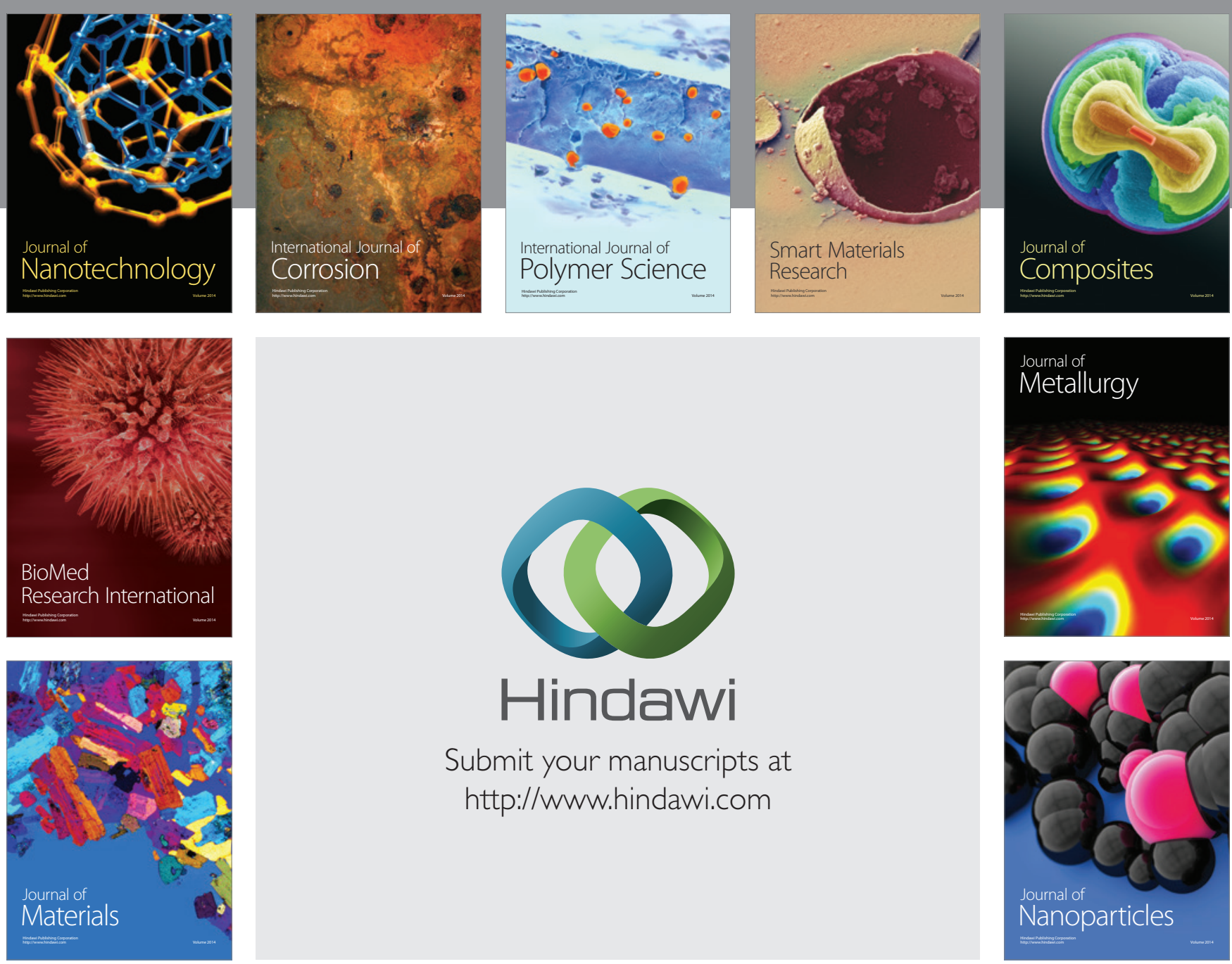

Submit your manuscripts at http://www.hindawi.com
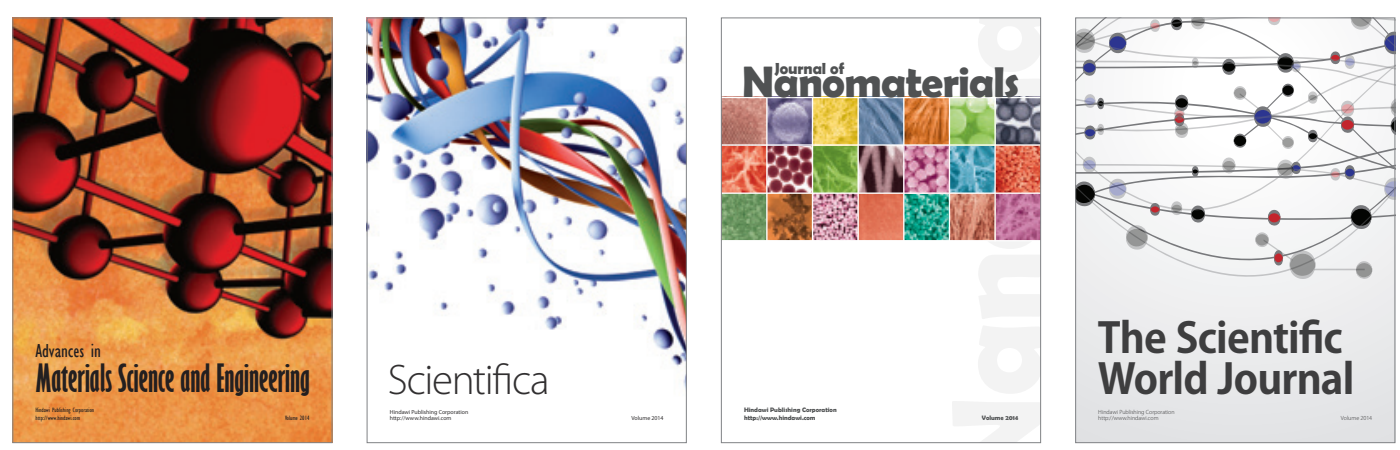

\section{The Scientific World Journal}
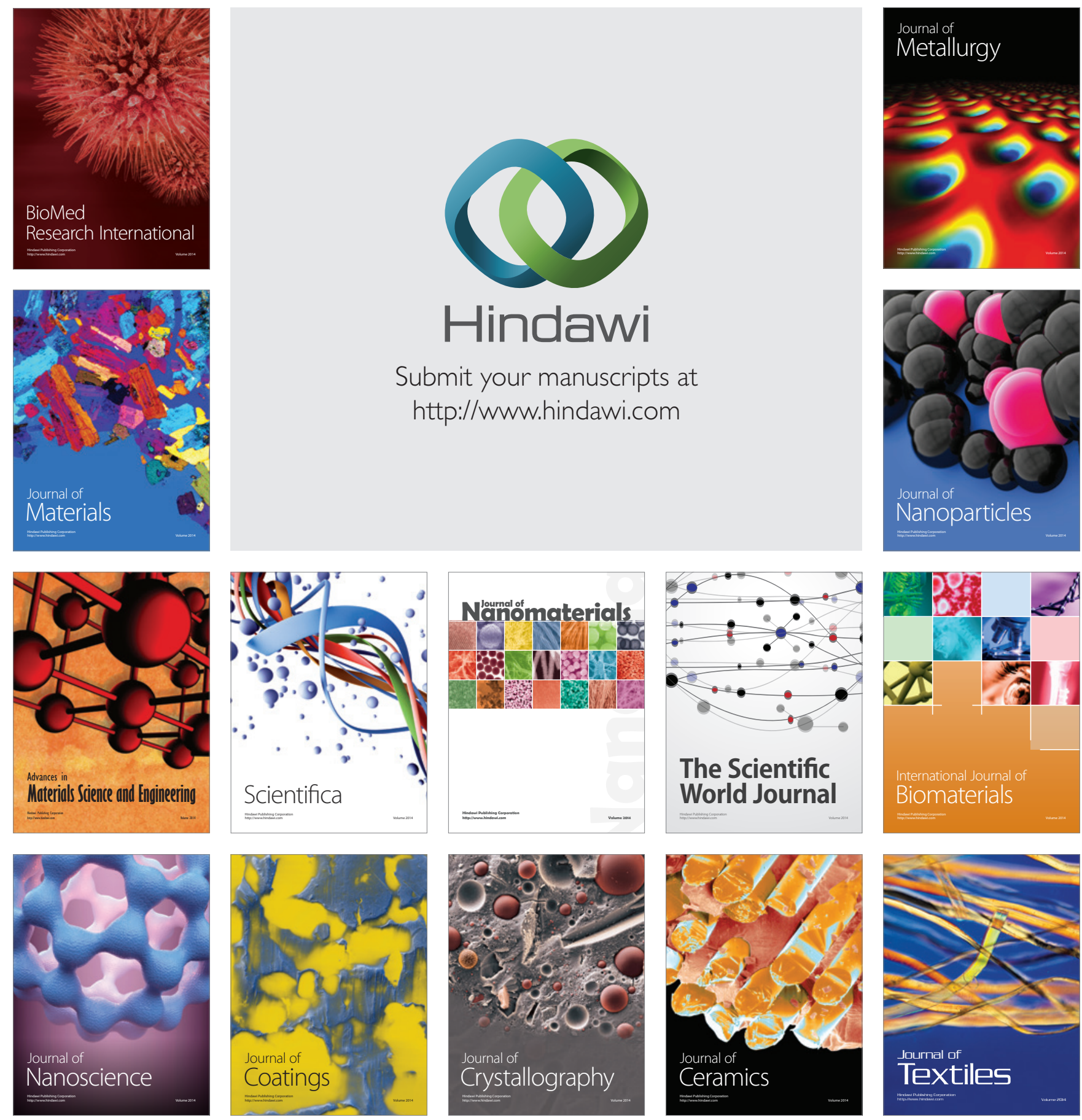